\title{
Two Novel Quantum-Dot Cellular Automata Full Adders
}

\author{
Mahdie Qanbari and Reza Sabbaghi-Nadooshan \\ Department of Electrical Engineering, Islamic Azad University Central Tehran Branch, Tehran 1469669191, Iran \\ Correspondence should be addressed to Reza Sabbaghi-Nadooshan; r_sabbaghi@iauctb.ac.ir
}

Received 30 November 2012; Revised 28 March 2013; Accepted 5 April 2013

Academic Editor: Yuanxin Zhou

Copyright (c) 2013 M. Qanbari and R. Sabbaghi-Nadooshan. This is an open access article distributed under the Creative Commons Attribution License, which permits unrestricted use, distribution, and reproduction in any medium, provided the original work is properly cited.

Quantum-dot cellular automata (QCA) is an efficient technology to create computing devices. QCA is a suitable candidate for the next generation of digital systems. Full adders are the main member of computational systems because other operations can be implemented by adders. In this paper, two QCA full adders are introduced. The first one is implemented in one layer, and the second one is implemented in three layers. Five-input majority gate is used in both of them. These full adders are better than pervious designs in terms of area, delay, and complexity.

\section{Introduction}

Quantum-dot cellular automata (QCA) is a new nanotechnology that can help us to reach low-power consumption, high device density, and high clock frequency. QCA size is smaller than CMOS it can, even be implemented in molecule or atom size. QCA power consumption is extremely lower than CMOS because there are not any current in the circuit and output capacity. The two important gates in QCA are three-input majority gate and inverter. In this paper, two novel QCA full adders are introduced in which the first one is implemented in one layer and the second one is implemented in three layers. They have five-input majority gate in their structure.

The rest of the paper is as follows. Section 2 describes QCA. Section 3 describes five-input majority gates. Two new full adders are presented in Section 4. Finally, Section 5 concludes the paper.

\section{Quantum-Dot Cellular Automata (QCA)}

Quantum-dot cellular automata (QCA), first proposed in 1993 by Lent et al. [1], is a structure made up of identical cells realized through a variety of technologies such as electrodynamic, ferromagnetic, and molecular [2].

A QCA cell a square shape, with four quantum dots in which just two electrons can stand at it. The electrons perch diagonally in the square for getting the farthest position from each other because of coulombic repulsive force. When the two electrons are positioned as in Figure 1(a), the polarization is $(+1)$, and if they are positioned as in Figure $1(b)$, the polarization is $(-1)$.

A QCA wire can be built by placing a row of cells as shown in Figure 2 when the first cell's polarization is +1 , the other's polarization becomes +1 , and when the input cell's polarization is -1 , the other cell's polarization has the same value.

The most important gates in QCA are inverters and threeinput majority gates. The inverter is shown in Figure 3(a), when the input cell has a value, the output cell become reversed. A three-input majority gate is made of three inputs, one device cell and one output as shown in Figure 3(b). If most of inputs have polarization +1 , the device cell becomes +1 . If most of inputs have polarization -1 , the device cell becomes -1 . The output cell's polarization follows the device cell's polarization.

AND gates and OR gates can be implemented by threeinput majority gate. If one of the inputs be +1 , then the threeinput majority gate is like an OR gate (1). If one of the inputs be -1 , then the three-input majority gate is like an AND gate (2):

$$
\begin{aligned}
& \operatorname{Maj}(A, B, 1)=A+B \\
& \operatorname{Maj}(A, B, 0)=A \cdot B .
\end{aligned}
$$




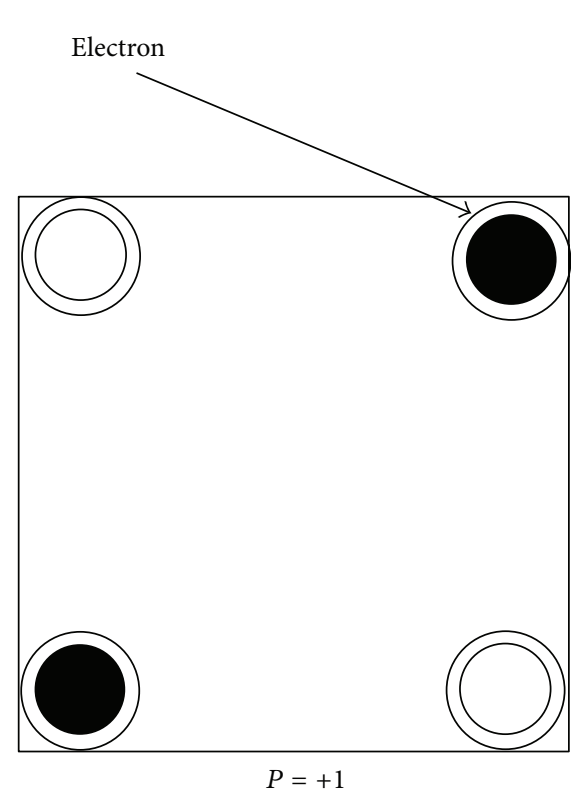

(a)

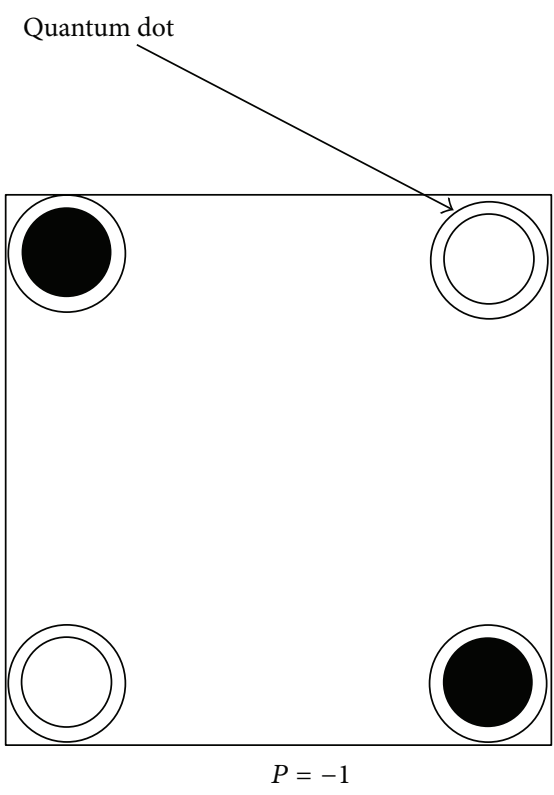

(b)

FIGURE 1: The polarization of cells.

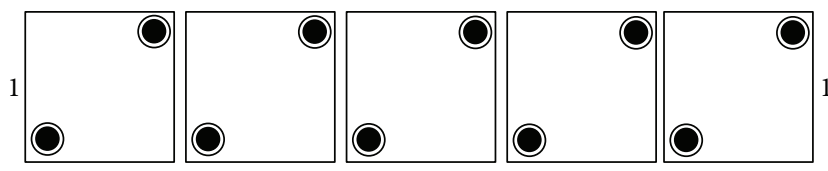

Figure 2: A QCA wire.

A QCA clocking zone is a group of QCA cells which is controlled by the same QCA clock. In QCA, four clocking zones are considered. The schematic of a QCA wire constructed using four clocking zones is shown in Figure 4(a). There is a $90^{\circ}$ phase delay from one clocking zone to the next one as shown in Figure 4(b) [6].

\section{Five-Input Majority Gates}

For reaching our goal, we need another gate and it is called five-input majority gates.

There are many five-input majority gates which were proposed from 2007 until now. As shown in Figure 5, Azghadi et al. [3], Navi et al. [4, 5], Akeela and Wagh [2], and Hashemi et al. [6] introduced some five-input majority gates. These five-input majority gates have some advantages and disadvantages.

We examined and found that the five-input majority gate, which was proposed in [2], is the best choice for our full adder, because it has ordinary cells, the inputs and outputs are not surrounded by the other cells, and they can be accessed easily. This design is suitable to implement larger QCA circuits, so we choose it for our full adder.

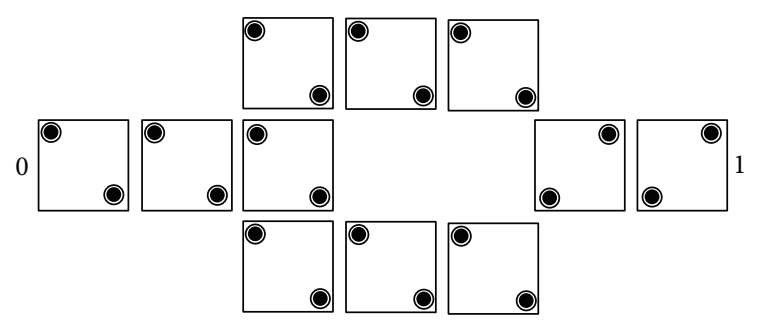

(a)

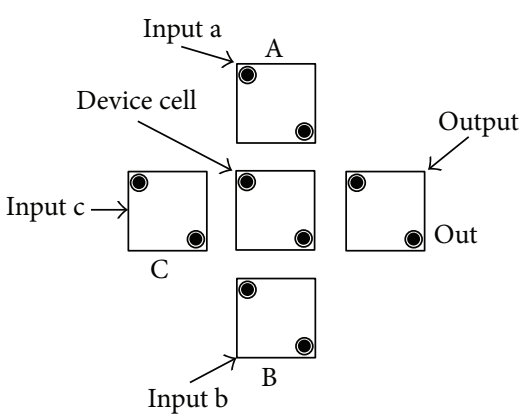

(b)

Figure 3: (a) A QCA inverter. (b) A QCA majority gate.

\section{Two Novel QCA Full Adders}

Many QCA full adders have been introduced until now. The first one is presented by Tougaw and Lent in 1994 [7]. As it is shown in Figure 6, it has five three-input majority gates and three inverters. This full adder uses QCA coplanar wire crossing scheme [6]. It is implemented in one layer, and it has 192 cells. In this design, QCA clocking concepts are not 


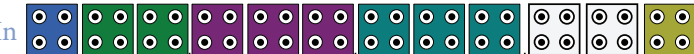
Zone 0
Zone 1
Zone 2
Zone 3

(a)

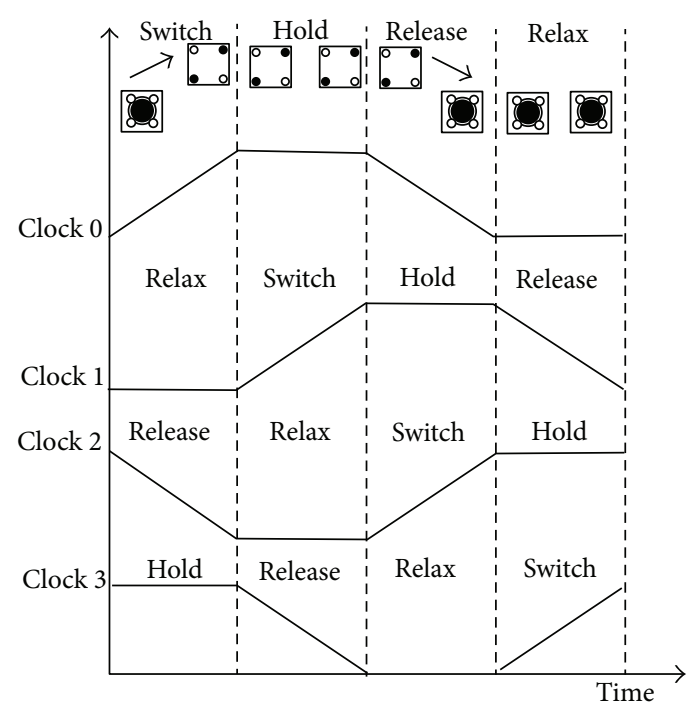

(b)

FIGURE 4: (a) A QCA wire with four clocking zones, (b) QCA clocks wave forms.

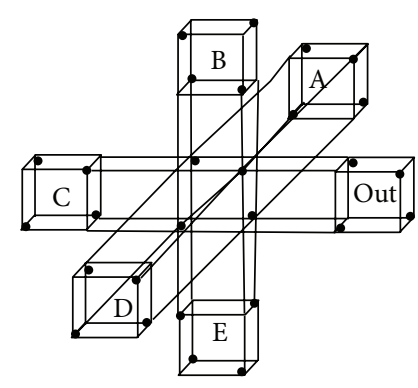

(a)

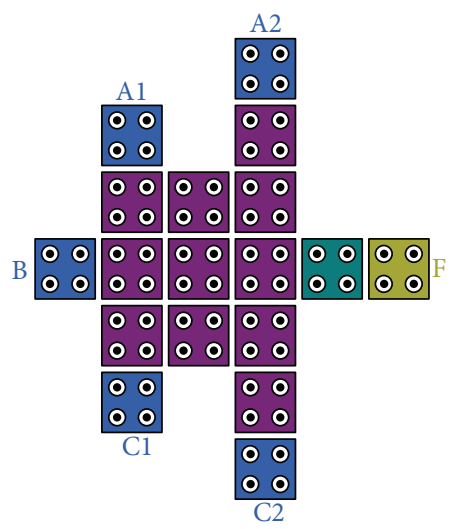

(d)

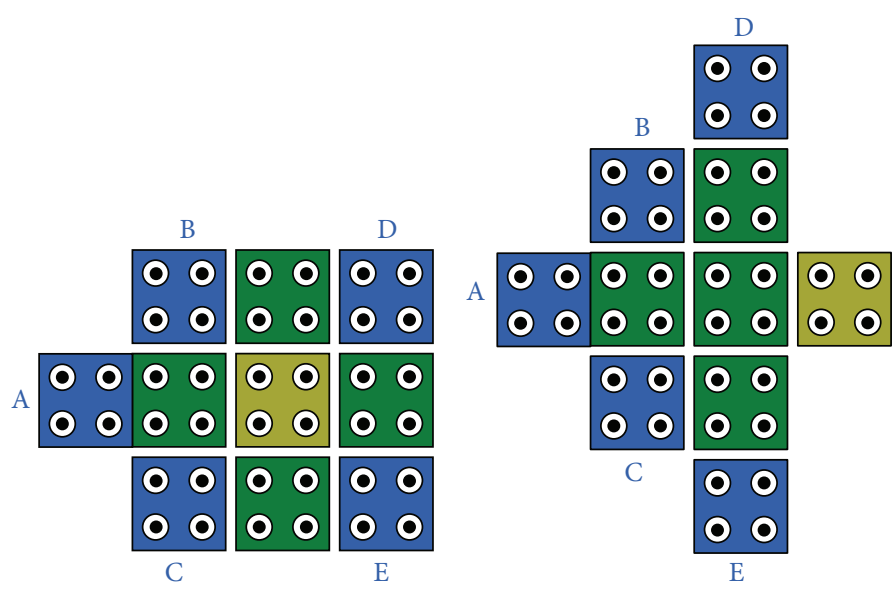

(b)

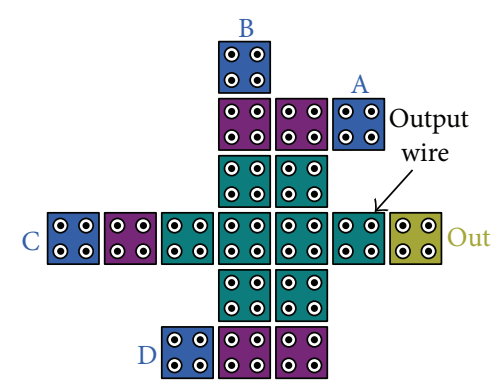

(e) (c)

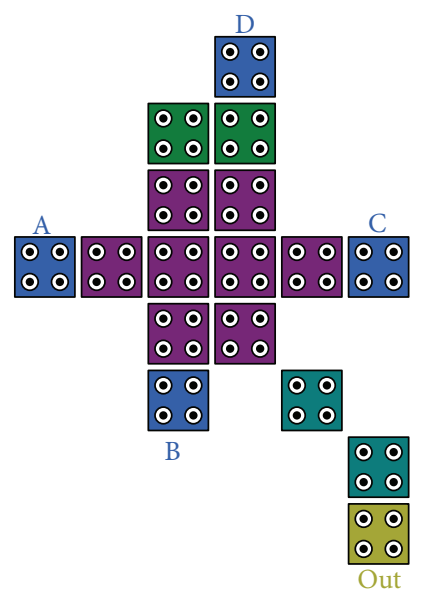

(f)

FIGURE 5: The proposed five-input majority gates in the literature ((a) [3], (b) [4], (c) [5], (d) [2], (e) [6], (f) [6]). 


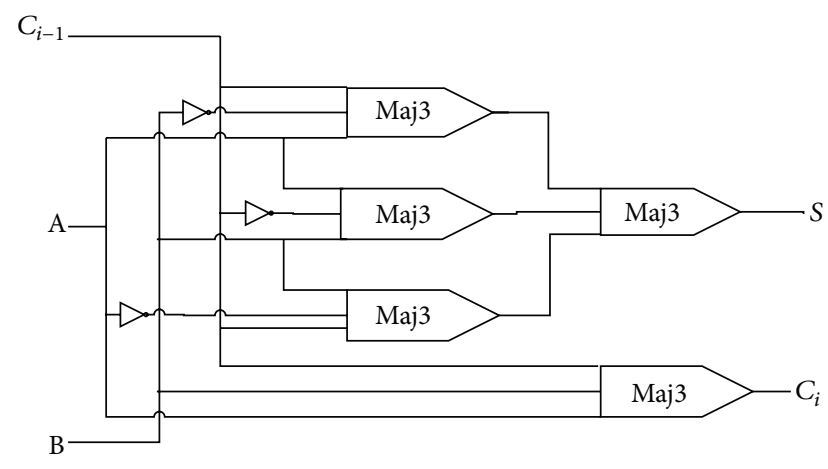

FIgURE 6: One-bit QCA full adder schematic [7].

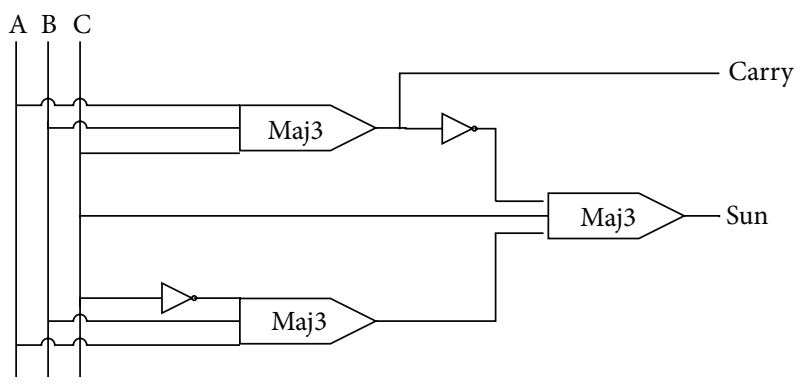

FIGURE 7: One-bit QCA full adder schematic [8].

considered [6]. Another QCA full adders using some logical structures is introduced by Vetteth et al. in 2002 [9], but the QCA clocking concepts are considered at it. For generating the output, it takes $3 / 5$ clock cycles. Another QCA full adder is introduced by Wang et al. in 2003 [8], which is made of three three-input majority gates and two inverters. It takes $1 / 25$ clock cycles to produce outputs. The schematic of this full adder is shown in Figure 7.

There are some QCA full adders that are implemented in three layers by Cho [10], Cho and Swartzlander [11, 12], Hänninen and Takala [13], Kim et al. [14], Zhang et al. [15], Navi et al. [4, 5], Sayedsalehi et al. [16], and Hashemi et al. [6].

In this paper, we proposed two QCA full adders in which the first one is implemented in one layer and the second one is implemented in three layers. In these designs, at first the carry value is calculated by a three-input majority gate (3), and then the carry value is inverted and is used as the two inputs of the five-input majority gate. The output of the five-input majority gate generates the some value:

$$
\begin{gathered}
\text { Carry = Maj3 (A0, B0, C0), } \\
\text { Sum } \left.=\text { Maj5 (A0, B0, C0, Carry' }{ }^{\prime} \text { Carry' }\right) .
\end{gathered}
$$

In this paper, two full adders were simulated by QCA designer software (version 2.0.3) [17]. The coherence vector computational engine was used in all simulations, and the important parameters are cell size $=18 \mathrm{~nm} \times 18 \mathrm{~nm}$, dot diameter $=5 \mathrm{~nm}$, and radius of effect $=40 \mathrm{~nm}$.

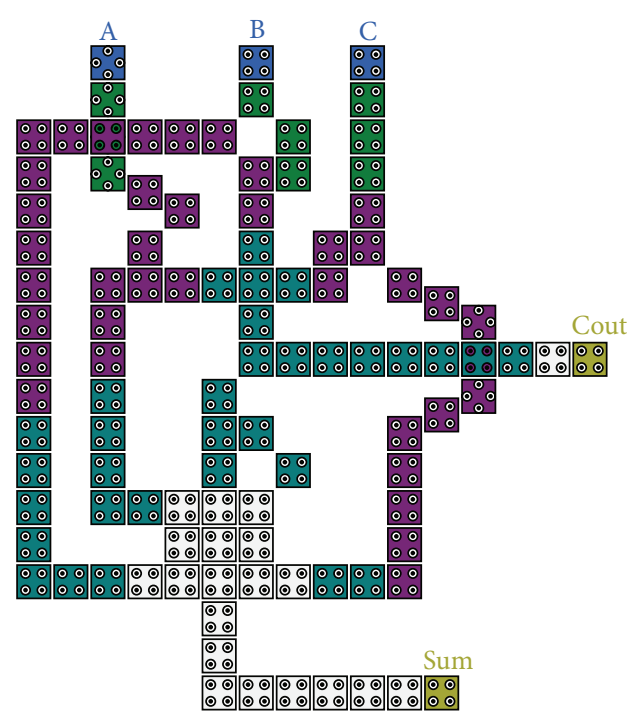

FIgURE 8: The first QCA full adder (it is implemented in one layer).

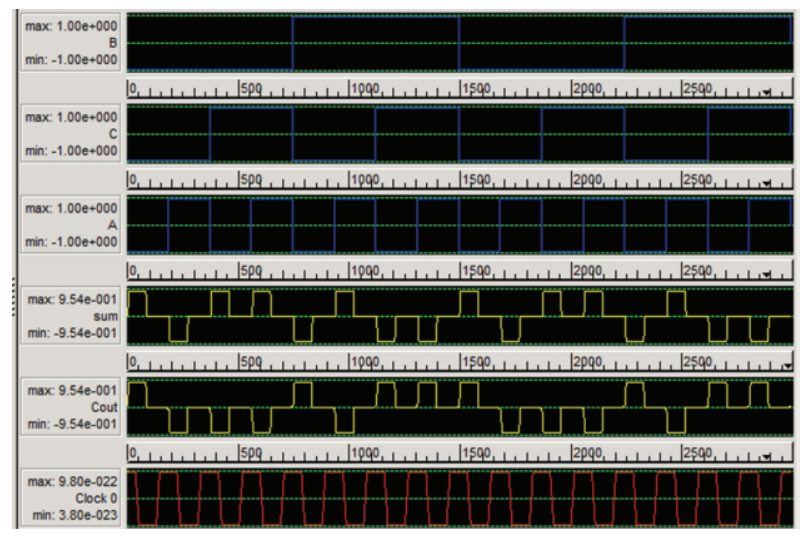

FIGURE 9: The simulation results of the first QCA full adder.

The first full adder uses QCA coplanar wire crossing scheme. As shown in Figure 8, this full adder takes one clock cycles to generate output. It has 104 QCA cells, and its area is $0.13 \mu \mathrm{m}^{2}$. The simulation result has been shown in Figure 9 .

The second full adder is implemented in three layers. This full adder takes 0.75 clock cycles to generate output as shown in Figure 10. It has 63 QCA cells and its area is $0.05 \mu \mathrm{m}^{2}$. In this full adder like the previous full adder, the inputs and outputs are not surrounded by the other cells and they can be accessed easily; the sum value and the carry out value come out from the same side, and they are suitable to implement larger QCA circuits. The simulation result has been shown in Figure 11 .

Table 1 compares our designs and the best previous designs. The first full adder is better than the previous designs which were implemented in one layer in terms of area, delay, and complexity. Also, the second one is better than the previous designs which were implemented in three layers in terms of delay and complexity. 
TABLE 1: The comparison between our proposed designs and the best previous designs.

\begin{tabular}{lcccc}
\hline QCA full adders & Layer's number & Complexity (cells) & Area $\left(\mu \mathrm{m}^{2}\right)$ & Latency (clk cycle) \\
\hline Coplanar QCA FA [8] & 1 & 145 & 0.17 & 1.25 \\
Proposed QCA FA in Figure 8 & 1 & 104 & 0.13 & 1 \\
Multilayer QCA FA [6] & 3 & 79 & 0.05 & 1.25 \\
Proposed QCA FA in Figure 10 & 3 & 63 & 0.05 & 0.75 \\
\hline
\end{tabular}

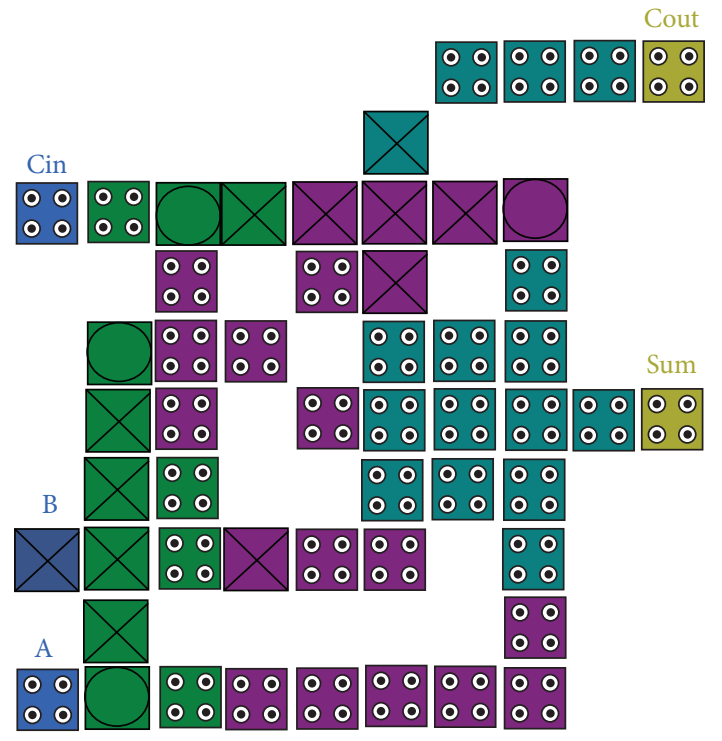

FIGURE 10: The second QCA full adder (it is implemented in three layers).

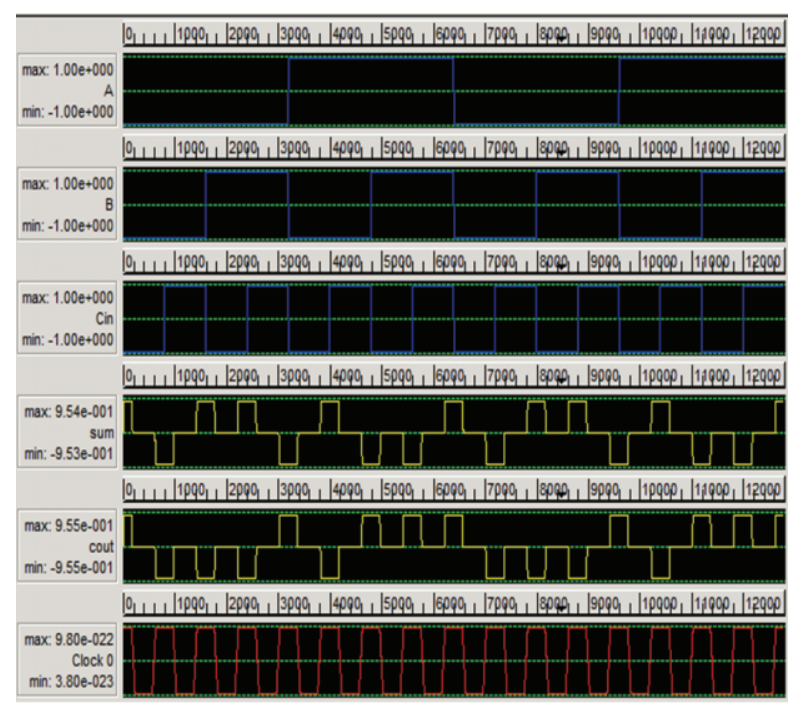

FIGURE 11: The simulation results of the second QCA full adder.

\section{Conclusions}

As mentioned earlier, low power consumption, high device density, and high clock frequency are the goal of QCA technology.
In this paper, two novel QCA full adders were proposed in which the first one is implemented in one layer and the second one is implemented in three layers. They have ordinary cells, and the inputs and outputs are not surrounded by the other cells and they can be accessed easily. The sum value and the carry out value come out from the same side and they are suitable to implement larger QCA circuits. The first full adder is better than the previous designs in terms of area, delay, and complexity. The second full adder is better than the previous designs in terms of delay and complexity but its area and the best previous designs area are equal.

\section{Conflict of Interests}

None of the authors have a direct financial relation with the commercial identity mentioned in the paper that might lead to a conflict of interests for the authors.

\section{References}

[1] C. S. Lent, P. D. Tougaw, W. Porod, and G. H. Bernstein, “Quantum cellular automata," Nanotechnology, vol. 4, no. 1, pp. 49-57, 1993.

[2] R. Akeela and M. Wagh, "A five input majority gate in quantumdot cellular automata," NSTI Nanotech, vol. 2, pp. 978-981, 2011.

[3] M. R. Azghadi, O. Kavehei, and K. Navi, "A novel design for quantum-dot cellular automata cells and full adders," Journal of Applied Sciences, vol. 7, no. 22, pp. 3460-3468, 2007.

[4] K. Navi, S. Sayedsalehi, R. Farazkish, and M. R. Azghadi, "Fiveinput majority gate, a new device for quantum-dot cellular automata, Journal of Computational and Theoretical Nanoscience, vol. 7, no. 8, pp. 1546-1553, 2010.

[5] K. Navi, R. Farazkish, S. Sayedsalehi, and M. R. Azghadi, "A new quantum-dot cellular automata full-adder," Microelectronics Journal, vol. 41, no. 12, pp. 820-826, 2010.

[6] S. Hashemi, M. Tehrani, and K. Navi, "An efficient quantum-dot cellular automata full-adder," Scientific Research and Essays, vol. 7, pp. 177-189, 2012.

[7] P. D. Tougaw and C. S. Lent, "Logical devices implemented using quantum cellular automata," Journal of Applied Physics, vol. 75, no. 3, pp. 1818-1825, 1994.

[8] W. Wang, K. Walus, and G. A. Jullien, "Quantum-dot cellular automata adders," in Proceedings of the 3rd IEEE Conference on Nanotechnology (IEEE-NANO'03), vol. 2, pp. 461-464, 2003.

[9] A. Vetteth, K. Walus, G. A. Jullien, and V. Dimitrov, "Quantum dot cellular automata carry-look-aheed adder and barrel shifter," in Proceedings of the IEEE Emerging Telecommunication Technologies, vol. 2, pp. 1-4, Dallas, Tex, USA, 2002. 
[10] H. Cho, Adder and multiplier design and analysis in quantumdot cellular automata [Ph.D. thesis], Faculty of the Graduate School, University of Texas, Austin, Tex, USA, 2006.

[11] H. Cho and E. E. Swartzlander, "Adder designs and analyses for quantum-dot cellular automata," IEEE Transactions on Nanotechnology, vol. 6, no. 3, pp. 374-383, 2007.

[12] H. Cho and E. E. Swartzlander, "Adder and multiplier design in quantum-dot cellular automata," IEEE Transactions on Computers, vol. 58, no. 6, pp. 721-727, 2009.

[13] I. Hänninen and J. Takala, "Binary adders on quantum-dot cellular automata," Journal of Signal Processing Systems, vol. 58, pp. 87-103, 2010.

[14] K. Kim, K. Wu, and R. Karri, "The robust QCA adder designs using composable QCA building blocks," IEEE Transactions on Computer-Aided Design of Integrated Circuits and Systems, vol. 26, no. 1, pp. 176-183, 2007.

[15] R. Zhang, K. Walus, W. Wang, and G. A. Jullien, "Performance comparison of quantum-dot cellular automata adders," in Proceedings of the IEEE International Symposium on Circuits and Systems (ISCAS '05), vol. 3, pp. 2522-2526, May 2005.

[16] S. Sayedsalehi, M. H. Moaiyeri, and K. Navi, "Novel efficient adder circuits for quantum-dot cellular automata," Journal of Computational and Theoretical Nanoscience, vol. 8, no. 9, pp. 1769-1775, 2011.

[17] K. Walus, T. J. Dysart, G. A. Jullien, and R. A. Budiman, "QCADesigner: a rapid design and simulation tool for quantum-dot cellular automata," IEEE Transactions on Nanotechnology, vol. 3, no. 1, pp. 26-31, 2004. 

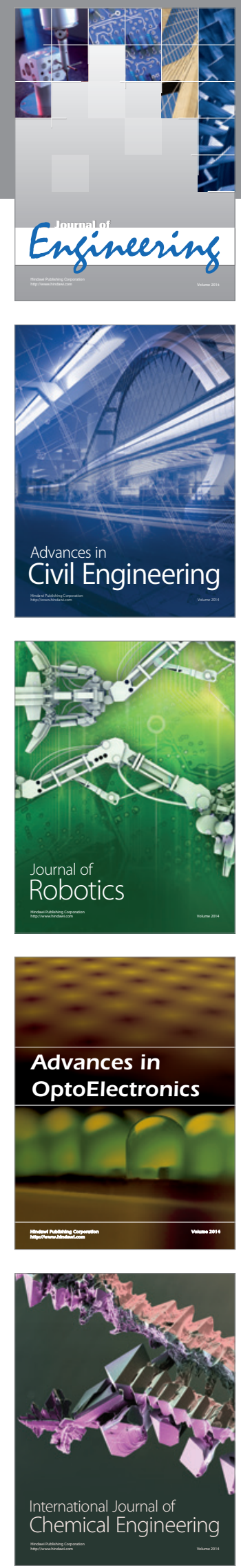

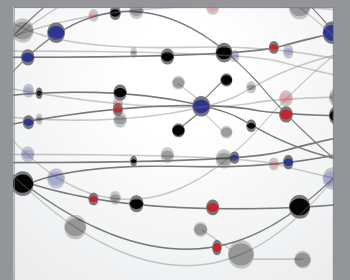

The Scientific World Journal
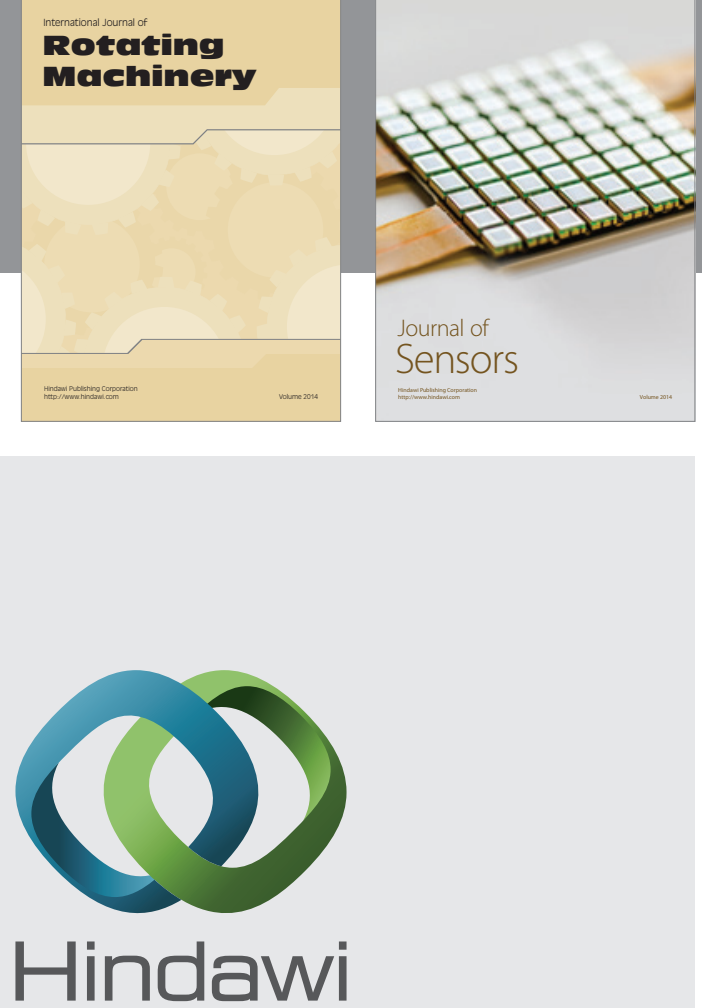

Submit your manuscripts at http://www.hindawi.com
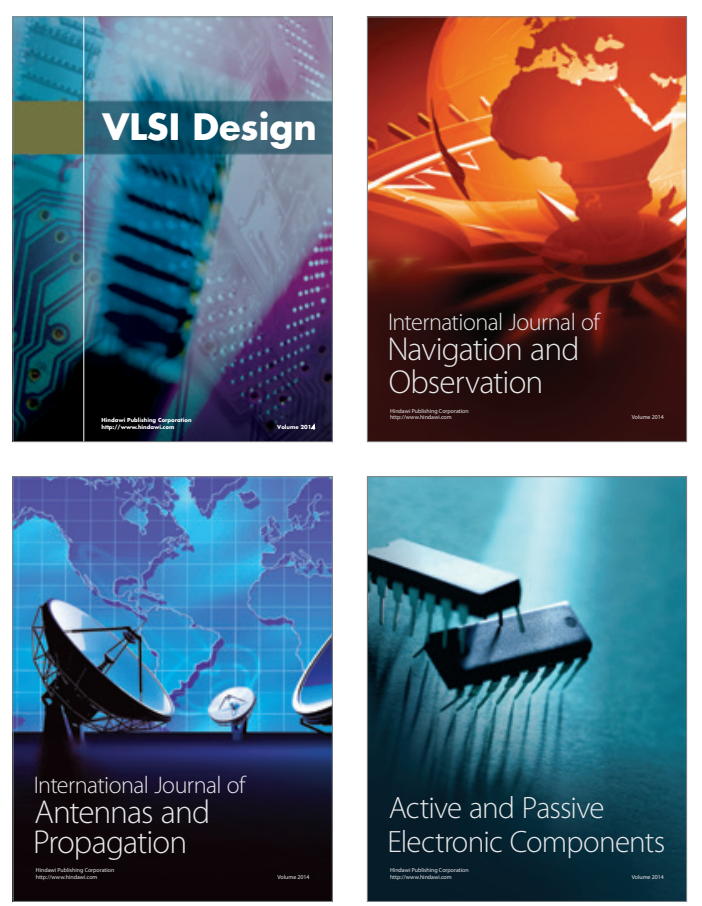
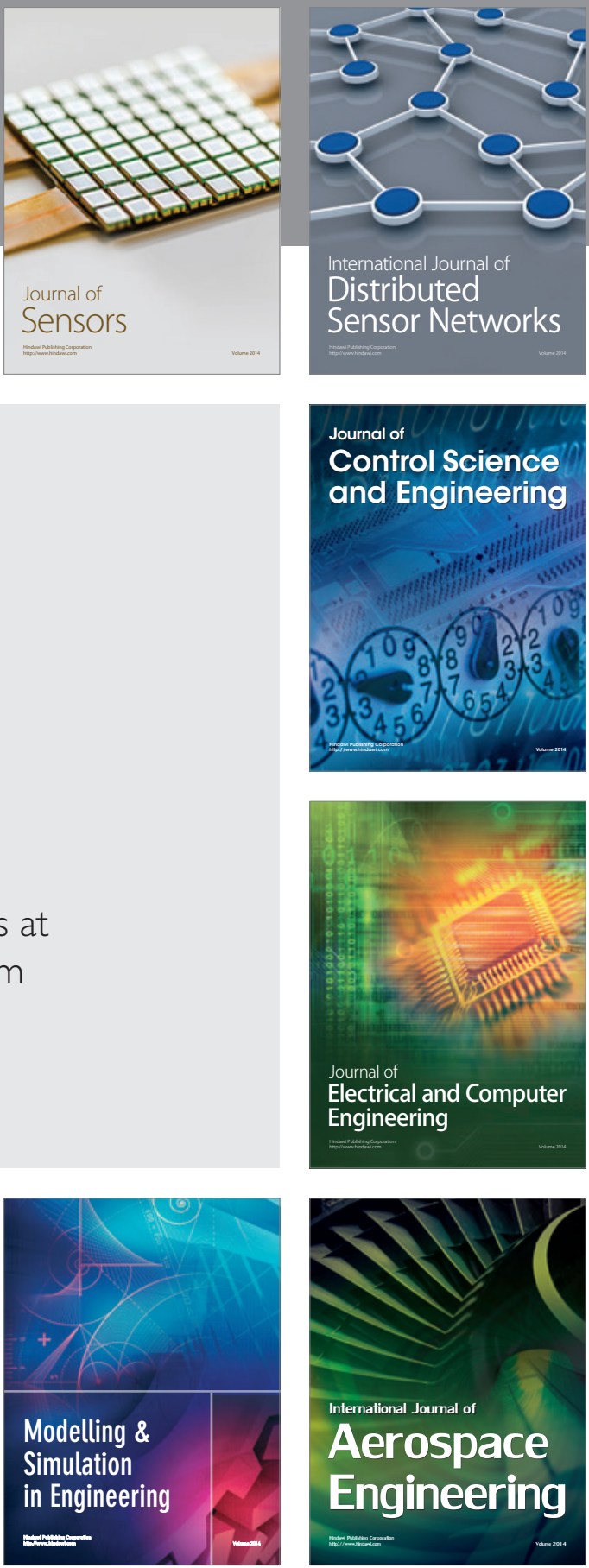

Journal of

Control Science

and Engineering
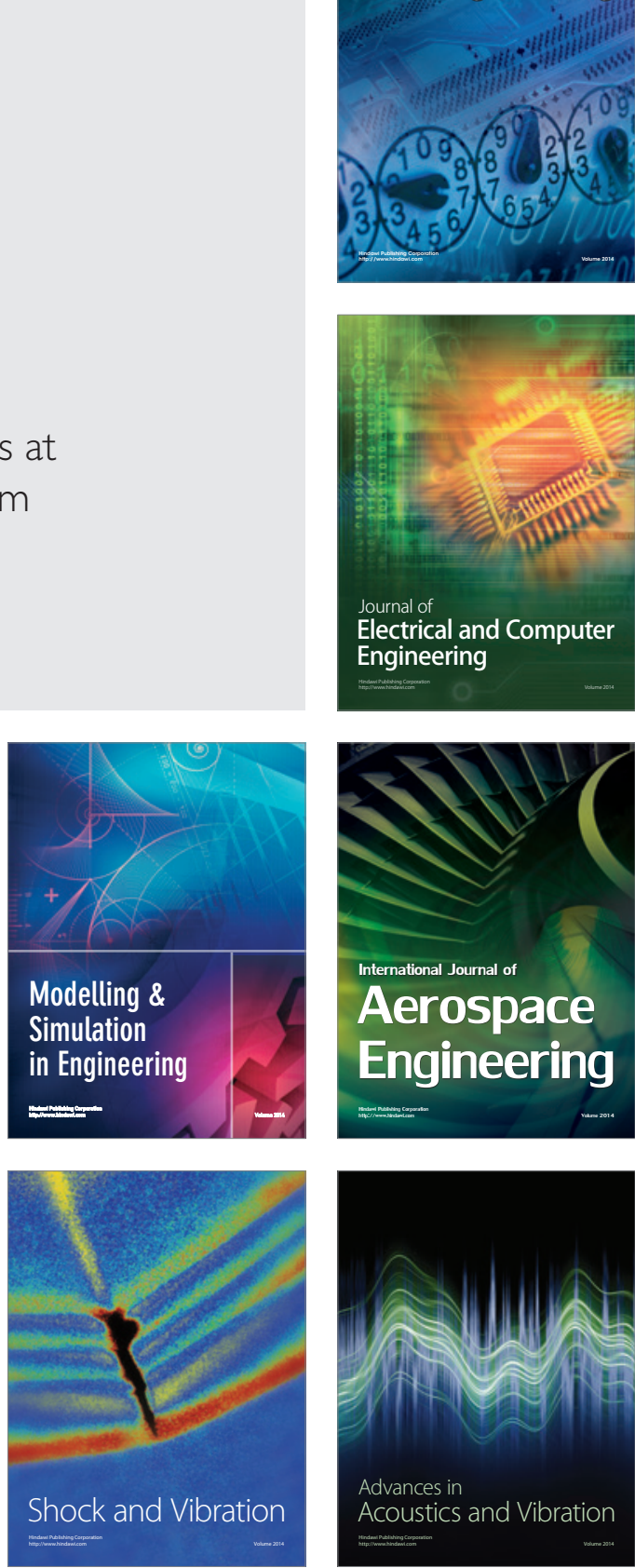